\title{
Aproximación al significado en la participación comunitaria. Estudio de caso en la población El Castillo en la diáspora del siglo XXI
}

\author{
Matías Prado ${ }^{1}$
}

Fecha de recepción: 27 de septiembre de 2014

Fecha de aprobación: 05 de noviembre de 2014

\section{Resumen}

El presente artículo se constituye a partir de un proceso de investigación, focalizado en la participación comunitaria en dos villas de la población El Castillo, perteneciente a la comuna de La Pintana en Santiago de Chile. La dinámica del artículo circula en torno a la asignación de significado de la participación en la comunidad. En el plano metodológico, muestra el desarrollo de una investigación en la que se ha utilizado el estudio de caso con el propósito de comprender, en mayor profundidad, los procesos participativos de las organizaciones, cuestión que ha dado cuenta de las estructuras significativas en la participación comunitaria.

Palabras clave: participación comunitaria, significado, acción social, estudio de caso, mundo de la vida.

\section{Approaching to significance in community involvement. Case study in el catillo settlement in the XXI century diaspora}

\begin{abstract}
This article was born from a research process focused on community participation in two villages of El Castillo settlement belonging to $\mathrm{La}$ Pintana municipality in Santiago, Chile. The dynamics circulates around the assignment of significance to community involvement. At methodological level, it shows the development of a research that has used the case study in order to understand in more depththe participatory processes of organizations, which has accounted for significant structures on community participation.
\end{abstract}

Keywords: Community participation, significance, social action, case study, life world 


\title{
Aproximação ao significado na participação comunitária. Estudo de caso em população o castillo na diáspora do século XXI
}

\begin{abstract}
Resumo
O presente artigo se constitui a partir de um processo de investigação focado na participação comunitária em duas vilas da população O Castillo, pertencente ao município da Pintana, em Santiago do Chile. A dinâmica do presente escrito circula em torno da atribuição de significado de participação na comunidade. Num nível metodológico, mostra-se o desenvolvimento de uma pesquisa na qual se tem utilizado o estudo de caso, a fim de compreender, com maior profundidade, os processos participativos das organizações. Questão que tem dado conta das estruturas significativas na participação comunitária.
\end{abstract}

Palavras-chave: Participação Comunitária, significado, ação social, estudo de caso, mundo da vida.

\section{Introducción}

A continuación se describe el camino que ha permitido acceder a los significados otorgados a la participación comunitaria en dos juntas de vecinos en la población El Castillo, ubicada en la comuna de La Pintana en la Región Metropolitana de Chile. El principal objetivo del artículo es que el lector comprenda los significados atribuidos a la participación en el territorio social y geográfico señalado.

La relevancia del estudio recae en la comprensión de los significados participativos para la construcción de políticas públicas orientadas al desarrollo sustentable de la comunidad.

Desde una perspectiva metodológica, se muestran las dificultades y ventajas a la hora de comprender los significados en la participación comunitaria actual. En el ámbito teórico, se ponen de manifiesto las principales categorías de significado en Weber (1997) y Schutz (1993), que subrayan una discusión necesaria en función de la comprensión de los significados actuales en los procesos de participación.

El proceso de comprensión de los significados inscritos en la participación de la organización vecinal, se encuentra caracterizado por darse mediante un estudio de caso por etapas, que dio 
cuenta del contexto significativo de la acción en la organización comunitaria, a través de un análisis estructural de la información obtenido en el trabajo de campo.

\section{El caso}

El Castillo es una población ubicada en la comuna de La Pintana, constituida mayoritariamente por pobladores que llegaron a la zona debido a un proceso de erradicación de campamentos en la década de los '80, a cargo del Ministerio de Vivienda de la época, y que consistió en la reubicación de familias que habitaban en las comunas de Santiago y Las Condes en sectores periféricos de la Región Metropolitana. Entre las comunas que fueron utilizadas como captadoras de las comunidades, muchas de estas disueltas, se encuentra la comuna de La Granja, la cual, debido a su tamaño, cedió una parte de su territorio para ser administrado por la naciente comuna de La Pintana.

Estos elementos históricos caracterizan a la población como comunidad, pues debemos entenderla como el conjunto de habitantes de un territorio, con historia, identidad, problemas y formas comunes de vivir. Lo cual se condice con la configuración de las villas Nueva Patagonia y El Remanso en la población El Castillo.

Parte de los elementos que permiten constituir y describir el contexto socioeconómico, sumado al histórico, de la población El Castillo, son: 1) la calidad de vida urbana; 2) la delincuencia, y 3) la drogadicción al interior de la comunidad. Bajo este contexto, se puede señalar, por ejemplo, que los temas de delincuencia y drogadicción se perciben como asuntos de interés público para los vecinos de la población. La encuesta efectuada por la Fundación Junto al Barrio (JAB), en 2011, menciona que el 15,5\% y el 24,8\% de la comunidad reconoce que la delincuencia y la drogadicción, respectivamente, son los problemas más preocupantes en el barrio.

Estos indicadores son elementos que han ido incidiendo en la forma en que se relacionan los vecinos. Así, el 75,5\% de los en- 
cuestados manifiesta que no puede confiar en sus vecinos, lo que ha generado una creciente disminución en los niveles de participación comunitaria dentro de las organizaciones territoriales. Así lo muestra la misma encuesta, en la que solo el $24,4 \%$ reconoce participar actualmente en la Junta de Vecinos del barrio; mientras que, al preguntar a los vecinos si en el pasado participaron en alguna organización, el 58,8\% señala que sí lo ha hecho. De este número, el 27,8\% reconoce que lo hizo en la Junta de Vecinos.

Estos porcentajes y consideraciones son algunos de los elementos que han llevado a realizar el presente estudio, preguntándose cuáles son los significados inscritos en la participación comunitaria de aquellos que aún pertenecen a la organización vecinal.

\section{Respecto de la participación comunitaria}

Los efectos que generan los bajos niveles de participación comunitaria en El Castillo han sido diversos, ejemplo de esto es la ocupación del espacio público por agentes que consumen o trafican drogas dentro de la población, o bien el tiempo que es ocupado en funciones productivas ${ }^{2}$ por parte de los integrantes de los núcleos familiares al interior de la comunidad.

Las dificultades para desarrollar un proceso de organización, en función de un proyecto comunitario, trae como consecuencia que los individuos de la población no sean los actores principales del desarrollo y la trasformación de su realidad.

Por "participación comunitaria" entenderemos un proceso mediante el cual se trasforma la realidad local. Dicha participación está compuesta, fundamentalmente, por la puesta en prácti-

\footnotetext{
2 La expresión "funciones productivas" no solo se refiere a las labores que cumple un sujeto en una empresa formal, sino que también a las que son necesarias en la administración del hogar o bien en el desempeño en organizaciones no formales para la estructura social, ya sea venta de droga, de "cachureos" u otras, que se encuentran caracterizadas por no estar regularizadas a través de un documento formal.
} 
ca de capitales que conserva la propia comunidad en función del desarrollo del territorio.

Ejemplo de lo anterior es el trabajo realizado por Toledo (2006), estudio en el cual observa este la participación comunitaria en la prevención del dengue, en Santiago de Cuba, durante el año 2000, con un abordaje desde la perspectiva de los diferentes actores sociales. El objetivo principal de dicho estudio fue "explorar las percepciones sobre la participación comunitaria en la prevención del dengue durante la investigación formativa de un proyecto comunitario" (Toledo, 2006, p. 39), en la cual se combinan las técnicas cuantitativas y cualitativas para abordar las opiniones de expertos en salud, líderes comunales y población escogida aleatoriamente.

El autor rescata el conocimiento que tiene la población respecto del dengue, con el objetivo de desarrollar un área de trabajo que permita combatir la enfermedad. Bajo esta lógica, en el estudio se comprende la participación comunitaria como: "un proceso social inherente a los grupos humanos que comparten necesidades, que participan en la identificación de las mismas y en la implementación y evaluación de estrategias para solucionarlas" (Toledo, 2006, p. 40).

En este sentido, la participación comunitaria es un proceso mediante el cual los actores son protagonistas de la construcción de soluciones colectivas a los problemas que existen en el territorio donde cohabitan. Ahora la pregunta se centra en comprender cuáles son los significados otorgados a esta participación comunitaria.

\section{Aproximación al significado}

El siguiente apartado se articula en función del diálogo que se construye, producto de la captación y comprensión del significado en la acción en Weber (1997) y Schütz (1993), siendo necesario antes advertir al lector desde qué espacio estamos situados.

Toda acción se encuentra inscrita en el mundo de la vida cotidiana. Esta es el área de la realidad en la que el sujeto puede 
intervenir y modificar, al mismo tiempo que las objetividades y sucesos que se encuentran en esta región limitan su libertad de acción. Solo el mundo de la vida cotidiana puede constituirse como un mundo, el cual, según Schütz y Luckmann, es "circundante, común y comunicativo. El mundo de la vida cotidiana es, por consiguiente, la realidad fundamental y eminentemente del hombre" (Schütz y Luckmann, 2003, p. 25).

En la actitud natural, el sujeto se encuentra en el mundo que presupone y considera real. De tal manera, el sujeto nació en el mundo de la vida cotidiana y reconoce que este existe antes de él.

Al estar inserto en el mundo de la vida cotidiana, presupongo, como bien señalan Schütz y Luckmann (2003), que:

Otros hombres también existen en este mundo mío, y, en verdad, no solo de manera corporal y entre otros objetos, sino más bien como dotados de una conciencia que es esencialmente igual a la mía. Así, desde el comienzo, mi mundo cotidiano no es mi mundo privado, sino más bien un mundo inter-subjetivo. (p. 26).

Por lo tanto, la estructura principal de la realidad del sujeto es compartida con otros, de tal manera que, hasta cierto punto, puede obtener conocimiento respecto de las experiencias de un otro.

Entenderemos entonces que los sujetos viven en un mundo mediado por los encuentros "cara a cara", en los que se tiende a representar actos verbales, mediante los cuales los sujetos manifiestan su visión del entorno. Por medio de estas relaciones cara a cara los participantes toman posiciones frente a un otro.

En principio señalaremos que, según Goffman, "hay un orden social donde la actividad de diferentes actores se integra en un todo coherente, permitiendo el desarrollo, consciente o inconsciente, de ciertos fines o funciones globales" (Goffman, 1991, p. 92).

En las situaciones de interacción conversacional, los actos que se integran en la dinámica de interacción son actos comuni- 
cacionales. "Todo mensaje emitido por uno de los participantes es suficientemente significativo y aceptable para los demás participantes" (Goffman, 1991, p. 92).

Pero, ¿qué se quiere decir con que el mensaje emitido sea significativo? O bien, ¿qué procesos están detrás de la atribución de significado al mensaje o la acción del sujeto?

Podemos partir señalando que la atribución de significado a la acción del sujeto es lo que permite distinguir la acción de la conducta. Bajo esta mirada, se puede identificar una serie de características de la acción social, entre ellas que "la acción social no es idéntica: a) ni una acción homogénea de muchos, b) ni la acción de alguien influido por la conducta de otros" (Weber, 1997, p. 19).

Weber (1997), al referirse a la conducta significativa, inevitablemente está haciendo mención a una conducta racional, es decir, a una conducta orientada a un sistema de fines individuales discretos, cuestión que Schütz (1993) aclarará señalando:

[Weber] dio ingenuamente por sentado los fenómenos significativos del mundo social como una cuestión de acuerdo intersubjetivo, exactamente de la misma manera en que todos nosotros suponemos, en la vida diaria, la existencia de un mundo externo sometido a leyes, que se adecua a los conceptos de nuestra comprensión. (p. 39).

Weber (1997), al distinguir entre los tipos de conductas orientadas racionalmente a fines o valores, la emocional y tradicional, está presuponiendo que el significado y el motivo son el mismo en una acción. Lo cual, según Schütz (1993), lo llevó a múltiples contradicciones, puesto que existe una diferenciación entre el significado de la acción y el nivel con el cual se capta el significado, esto debido a que la mayoría de las acciones de los sujetos tienen un verdadero significado cuando son aisladas del flujo de experiencia y son consideradas.

Este ejercicio es observable en el siguiente extracto de entrevista realizada a la presidenta de la junta de vecinos Nueva Pata- 
gonia (2012), en la cual la entrevistada aísla una experiencia del flujo del mundo de la vida y la considera con el fin de otorgarle un significado al valor de su participación.

¡Ah, esa persona es así! ¡Esa persona es sinvergüenza! Entonces, al final abarcaban a todos, porque todos estábamos ahí, pero no todos somos iguales, no todos somos sinvergüenzas, porque para eso uno trabaja y yo, de mi bolsillo, de repente, he hecho comida, de mi bolsillo. (Presidenta (mujer), Nueva Patagonia, entrevista).

Por lo tanto, para Schütz (1993) es equívoco utilizar el criterio de significatividad de forma amplia, ya que esto lleva a la confusión al momento de distinguir la acción social de la meramente activa. De esta manera se logra señalar que, independiente de que la conducta sea tradicional o afectual, tiene algún nivel de significado, desterrando la posibilidad de que la diferenciación entre acción y conducta esté dada por la significación subjetiva o no.

En tanto, para Talcott Parsons (1984) ha quedado ausente la idea del orden en la teoría de la acción propuesta por Weber. Pues ha de señalar que privilegia las conductas racionales, dejando de lado las dimensiones no instrumentales de la acción social; "ya que para aquél la estructuración de la acción social no puede darse fuera de una matriz general de la orientación de las acciones" (Lutz, 2010, p. 210). Para esto Parsons (1968) señala que:

La acción puede, dice, estar orientada en términos del: a) uso (Brauch), b) interés (interessemlage), c) orden legítimo. El interés es la categoría en la que las uniformidades son comprensibles en términos de la orientación racional (zweckrational) de los actores hacia expectativas análogas. El concepto de orden legítimo, por otra parte, implica orientación de la acción hacia la idea (Vorstellung), por otra parte los actores, de la existencia de tal orden como norma. (p. 794). 
Lo que ha intentado hacer Parsons (1968) es defender las perspectivas voluntaristas de la acción, en las que el actor manifiesta la intencionalidad de alcanzar sus objetivos mediante su intervención en el medio material y social.

A lo que Weber (1997) podría contraargumentar señalando que las relaciones sociales tienen características más dinámicas y menos estructuradas, comprendiendo así el significado desde dos dimensiones: a) La primera hace referencia al significado subjetivo, el cual puede darse de manera observacional, es decir, el significado de la acción de otro se da mediante la observación directa de la acción. b) La segunda hace referencia a la trama más amplia de significado que se da en la acción. Este contexto es develado por la comprensión motivacional o esclarecedora.

Según Schütz (1993), Weber, al señalar que:

Comprendo mediante observación directa el significado de la conducta de un hombre cuando lo veo realizar actos tales como cortar madera (...), menciona estos movimientos observados del cuerpo de la otra persona como el substrato de la comprensión observacional. (p. 56).

Entonces, según Schütz (1993), Weber se ha equivocado porque, ¿qué sucede si el hombre con el hacha no va a cortar madera y solo parece hacerlo? Schütz (1993) concluye que la comprensión observacional de la conducta de un otro no es suficiente para saber qué es lo que pretende hacer el hombre con el hacha. Para esto se observa primero la conducta corporal y, de forma posterior, se ubica en un contexto de significado -que no es idéntico al contexto significativo del actor-, al cual llamaría "contexto objetivo de significado, por oposición al contexto subjetivo de significado del actor". (Schütz, 1993, p. 57).

A continuación, un extracto de entrevista a un participante de la Junta de Vecinos Nueva Patagonia (2012), que muestra cómo este vecino se ubica en un área del significado en la que su contexto subjetivo de significado de participación no es idéntico al contexto objetivo de significado de participación que presentan 
otros actores. "la gente que estaba no era confiable, po', no te daban la confianza" (Participante (hombre), Nueva Patagonia; entrevista).

Para Weber (1997), la motivación consiste en la comprensión del contexto del significado a la que pertenece la acción, una vez que se ha entendido el significado subjetivo que existe en la acción. Donde el contexto significativo es una parte apropiada de nuestro punto de vista. A lo que Schütz (1993) señalaría que no existe ninguna manera de saber si el contexto significativo apropiado para mí es el mismo que considera apropiado el actor que realiza la acción; por lo tanto, para comprender la motivación de un actor es necesario conocer aspectos de su pasado y su futuro.

El conocimiento del pasado de los sujetos que participan de la acción es necesario para que el yo pueda encontrar un contexto significativo en el cual ubicar las acciones. Mientras que el conocimiento del futuro de los sujetos que participan es necesario para determinar, según Schütz, "si sus acciones, en el sentido subjetivo que éstas tienen para ellos, resultan adecuadas al contexto significativo que ya he reconocido" (Schütz, 1993, p. 57).

Una cosa es la motivación, pero otra es el motivo de la acción. Y aquí Weber (1997) lo comprende como una relación de significados que al actor o el observador les parece un fundamento apropiado para justificar su conducta, es decir, la distinción entre el significado subjetivo y objetivo de una acción.

Schütz (1993) argumenta que Weber ha sido incapaz de contestar la pregunta sobre si el significado de la acción en un actor es idéntico al de su motivación. En definitiva, según Schütz, "el motivo de una acción no puede comprenderse a menos que se conozca primero el significado de la acción. Pero es el actor quien tiene ese conocimiento, no el observador" (Schütz, 1993, p. 59).

La pregunta que se suscita, en función del último párrafo, hace referencia a qué es el significado en la acción. Respecto de esto, se puede mencionar, en una primera instancia, que tiene relación con un acto ya constituido, o bien, una acción en curso que 
se constituye y que, por lo tanto, da paso a una serie de sucesos. De esta manera, Schütz (1993) señala:

Mi acción, tal como ocurre, se me presenta como una serie de vivencias existentes y presentes, vivencias que nacen y mueren. La acción que atiendo (interdientes) se me presenta como una serie de vivencias futuras. Mi acto terminado, completado (que es mi acción luego de expirada), se me presenta como una serie de vivencias terminadas que contemplo en mi memoria. (pp. 68-69).

Por lo tanto, el significado de la acción de cada actor no solo es la vivencia de la conciencia mientras la acción está en curso, sino que también las vivencias futuras y pasadas que constituyen la acción que atiendo y completo, respectivamente.

Ejemplo de lo que señala Schütz (1993) es lo mencionado por la presidenta de la Junta de Vecinos Nueva Patagonia (2012), donde se presenta un significado en la participación, dado por elementos precedentes que se proyectan con una intencionalidad de futuro. "Poder cambiar todos este sistema, este, porque aquí no se estaba trabajando con Junta de Vecinos, nadie se moviliza (...). Y quiero luchar, quiero luchar por muchas cosas que aquí en la población no están hechas" (Presidenta (mujer), Nueva Patagonia; entrevista).

El significado es captado por el actor mediante la selección de experiencias, convirtiéndolas en experiencias discretas, lo que influye en agentes que pueden ser contemporáneos, predecesores o sucesores.

En cuanto a los contemporáneos, son sujetos que coexisten conmigo y su duración es simultánea a la mía, pero aunque viva entre ellos no capto de forma directa sus experiencias, sino que, como señala Schütz, "infiero sobre la base de evidencia directa las vivencias típicas que deben tener (...), puedo no sólo observar sino también actuar, hacer que la conducta y las vivencias de mis contemporáneos sean motivo para mi acción" (Schütz, 1993, p. 172-173). 
Además del mundo de los contemporáneos, se logra reconocer el mundo de los predecesores, de los cuales solo se puede ser un observador y no un actor, ya que se constituyen antes de la existencia del sujeto. Mientras que también se me da el mundo de los sucesores, este mundo será habitado cuando el sujeto ya no exista. Como señala Schütz, "hombres de los cuales no sé nada como individuos y con cuyas vivencias no puedo tener contacto personal" (Schütz, 1993, p. 173). De hecho, únicamente se logra conocer las vivencias suponiendo que son similares a las de los contemporáneos y predecesores. Este mundo no puede ser vivenciado directamente y solo es captado de manera vaga.

\section{Metodología}

Los argumentos del artículo se constituyen a partir de un proceso de investigación mediante un estudio descriptivo e interpretativo de caso, lo que significó presentar un informe del objeto de estudio y utilizar los datos descriptivos para desarrollar categorías, con el fin de desafiar o defender la teoría.

Este tipo de estudio facilitó la comprensión de los significados de la participación en las organizaciones vecinales de las poblaciones Nueva Patagonia y El Remanso, en El Castillo, los cuales fueron los casos seleccionados, de manera de analizar el fenómeno de forma penetrante, es decir, lograr conocer la administración, problemas, construcción de redes de apoyo, formas de organización, motivaciones, historia de la organización, espacios de reunión y participantes.

El estudio consistió en tres etapas (Pérez, 1998): preactiva, interactiva y posactiva. En la primera fue necesario reconocer cuáles eran los elementos que guiarían el caso, es decir, los objetivos que se buscaban en el estudio. De manera complementaria, se buscó la información relevante, ya fueran estudios previos o información que mostrara una fotografía del contexto en el que se inscribe la organización vecinal, que facilitara la contextualiza- 
ción del caso y que posibilitara establecer los criterios de selección del mismo.

Las características de los informantes se establecen en esta etapa, y estos corresponden a dos modelos tipos: el primero hace referencia al sujeto participante o ex participante de la organización vecinal, sin un cargo específico en la misma, el cual, en su dimensión social y económica, se conduce de forma similar al resto de la comunidad, destacándose por ser agente que permite identificar la historia de la colectividad, procesos de deconstrucción y objetivos particulares o colectivos mediante su participación.

El segundo modelo tipo hace referencia a dirigentes de las unidades vecinales, quienes lograron dar un diagnóstico del nivel de inserción actual de los vecinos en temáticas comunitarias, cuestión que facilitó la identificación de los significados asignados a la participación comunitaria en el territorio.

La segunda fase del trabajo de campo consistió en la generación de confianzas entre el investigador y los dirigentes.

Los lazos de confianza se establecen desde la construcción de una red de colaboración, en la que el investigador se ubica como un agente facilitador y articulador de herramientas que permitan a la organización vecinal gestionar recursos económicos y no económicos para los objetivos que esta presenta; ejemplo de esto es la elaboración de una "carta"3.

En la tercera etapa se comparten los primeros resultados de la investigación, de forma diferenciada con cada participante. Esto debido, principalmente, a que los elementos que no fueron identificados son puntuales según cada sujeto.

En este momento de la investigación se accede a los informantes que dieron cuenta de categorías lingüísticas y culturales que no

3 La "carta" es una herramienta burocrática, presente en la cultura de la comunidad, como elemento que otorga la posibilidad de gestión de subsidio por parte de privados, particulares y / o entidades públicas en las actividades realizadas por la unidad vecinal. 
se lograron dilucidar en la primera etapa de entrevista, cuestión que posibilita despejar dudas y profundizar en otros elementos.

Una vez concluida la retroalimentación, se pasa al proceso de análisis, que facilita la elaboración de los primeros resultados y reflexiones.

Para el análisis de la información entregada, se utilizó el método de análisis estructural de contenido. La relevancia de este enfoque permitió poner énfasis en la construcción del significado en la participación comunitaria.

Este significado se produce en un contexto y modelo sociocultural específico. Por tanto, el propósito de este tipo de análisis $^{4}$ fue construir y describir en el discurso de los informantes las unidades que configuran los significados, de manera que en una segunda instancia se logre identificar la relación y dinámica en un contexto comunicativo.

A partir del propio discurso de los entrevistados, se logra la construcción de cruces axiales, orientadas a la identificación de cuatro tipos posibles de realidades. Donde $\mathrm{Y}$ - tiene su opuesto $(\mathrm{Y}+)$ y $\mathrm{X}+$ tiene su opuesto $(\mathrm{X}-)$. De esta manera, se construyeron los cuatro tipos de realidades identificadas por la población. Este proceso da cabida al análisis final y las debidas conclusiones que se presentan en este estudio.

Ejemplo:

\begin{tabular}{l|r}
\multicolumn{2}{c}{ Y+ } \\
Realidad $^{5}$ A & Realidad B \\
Realidad C & Realidad D \\
Y- $_{-}$
\end{tabular}

Para profundizar en el desarrollo del análisis estructural de contenido, se recomienda al lector, revisar a Martinic (1992).

5 En el caso de que alguno de los cuatro tipos de realidad quede en incógnita querrá decir que, a pesar de la posibilidad de su existencia en las distinciones y categorías realizadas en las entrevistas, no figura como una realidad manifiesta. 


\section{Vida y organización en espacios comunes}

A continuación se presenta el cruce axial "Significados de la participación", que da cuenta de cuatro realidades en las organizaciones vecinales de las villas Nueva Patagonia y El Remanso en la población El Castillo, expresando los principales significados en la participación comunitaria.

Luego se describe la realidad B y, posteriormente, se muestran los principales resultados que dan cuenta del significado atribuido a la participación comunitaria.

Cruce Axial: Significados de la participación

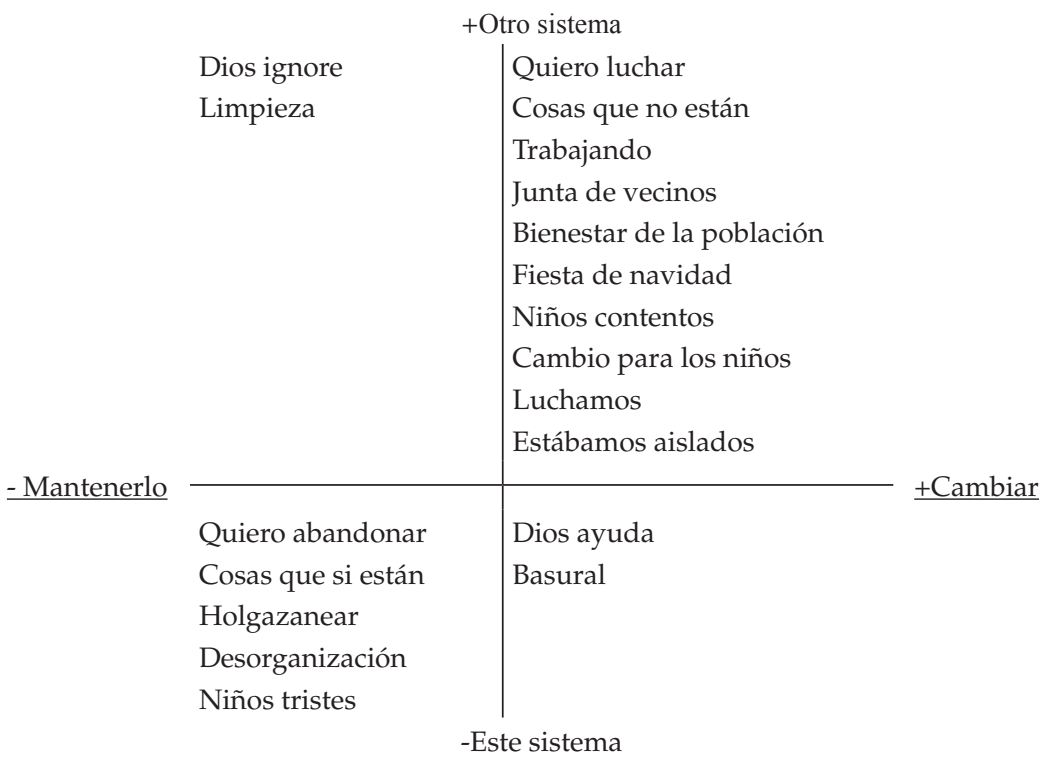

La realidad B se encuentra caracterizada por "Otro sistema" y "Cambiar". Las posibilidades de modificar las condiciones de vida en las cuales subsiste la población son, en esta realidad, las que articulan la participación, "tratar de luchar por el bienestar de la población no más, po'” (Participante (hombre), Nueva Patagonia; entrevista).

La protección a la niñez y "niños más contentos" se transforman en elementos fundamentales en la realidad B, la cual busca 
cambiar las condiciones actuales de la comunidad. Esto se intenta hacer mediante una lucha que permita alcanzar cosas significativas para la población, como luminarias y áreas verdes. Este propósito en la realidad B se pretende conseguir por medio del trabajo en conjunto con la Junta de Vecinos.

Estos elementos dan cuenta de un contexto socioespacial y temporal, que permite identificar los aspectos que construyen la columna vertebral de la participación comunitaria en el territorio.

\section{Configuración de la participación}

El contexto significativo de la participación se constituye a partir de un proceso de ruptura del primer espacio comunitario (campamento), caracterizado por la dispersión centrífuga de sus componentes a distintas zonas físicas de la región.

El traslado socioespacial que vive la población compone un nuevo espacio comunitario, caracterizado por configurarse a partir de historias y aspiraciones comunes, lo que les facilita desarrollar un trabajo focalizado en la mejora de las condiciones de vida en el territorio.

De esta manera, se conforman como una comunidad, definida por la comunión de sentimientos y aspiraciones de grupos particulares, unidos por su historia u origen. Es así como el segundo espacio comunitario requiere suplir necesidades básicas que, producto de su composición periférica, se ven mermadas, dando origen a las primeras áreas participativas, en las que la movilización de recursos materiales y simbólicos es esencial.

A partir de este proceso, la participación se encuentra orientada en una otredad, constituida a partir de los sentidos subjetivos que se le otorga a un "otro" como colectividad -o bien como individualidad- en el mundo de la vida cotidiana, mediante la significación del mundo por parte del sujeto. 
De este modo, el contexto significativo se encuentra ubicado en la configuración del espacio sociofísico en el que se constituyen las relaciones, las cuales son procesos experienciales comunes que los vinculan en función de la evaluación y modificación de la realidad local que comparten, es decir, se constituye un contexto significativo común. Por lo tanto, los motivos de la participación se encuentran ubicados como el resultado de una experiencia pasada, que se ha orientado a modo de un elemento causal precedente.

A través de este proceso, el significado de la acción se ha constituido mediante el devenir de la participación; es decir, en la medida en que un sujeto ha participado o ha enfrentado dificultades que lo han llevado hacia la organización, le ha atribuido un significado a su acción mediante la selección de la experiencia discreta. De esa forma, esta es significativa para el individuo que la ejecuta.

Así, el significado de la participación en la organización vecinal se encuentra ubicado en dos dimensiones elementales en la comunidad, las cuales paso a describir.

\section{Primera dimensión del significado}

Como se ha señalado, el contexto significativo se encuentra ubicado en la configuración del espacio sociofísico en el cual se constituyen las relaciones cara a cara. Estas se construyen a partir de procesos experienciales comunes, que los vinculan en función de la evaluación y modificación de la realidad local que comparten.

Por eso la importancia de que el primer aspecto significativo haga referencia a la conservación de la cultura del segundo espacio comunitario, el cual se ha construido a partir de los procesos de erradicación de campamentos.

Este segundo espacio comunitario se ha caracterizado por sostener dificultades propias de habitar en una zona periférica 
de la ciudad, carente de servicios básicos, oportunidades laborales y una penetración latente de la delincuencia. Así lo hace saber un entrevistado de la junta de vecinos El Remanso: "hoy día es medio complicado, o sea es que la droga... hay harto domestiquero, hay harto cabro que se mete a robar a las casas" (Participante (hombre), El Remanso; entrevista).

Producto de las condiciones en las cuales se instala el segundo espacio comunitario, la colectividad se ve en la necesidad de poner en ejercicio participativo sus recursos y capitales en función de la mantención de la comunidad instalada y de sus partes. Esta ejecución de recursos ha dado paso, de manera integradora, a una atribución significativa de la participación comunitaria, la que se manifiesta en el desarrollo de líneas de acción que se focalizan en el progreso del territorio, ya sea en áreas ambientales, económicas, de infraestructura o culturales.

Bajo esta lógica, el significado atribuido a la participación, en la primera dimensión, se encuentra asignado a las posibilidades existentes de mantener condiciones básicas de vida dentro de la comunidad, a la vez que se conservan los ritos asignados a la ejecución de los objetivos presentes en la organización vecinal.

\section{Segunda dimensión del significado}

El segundo aspecto en el que se encuentra ubicado el significado de la participación, hace referencia a los parámetros de comportamiento que se sostienen en la cultura, los cuales, mediante la ejecución de recursos que se orientan hacia los sucesores y contemporáneos, expresados en la niñez, conservan las reglas de la comunidad $^{6}$.

Bien se preguntaría Salazar (2006): ¿cómo es que repercute la vida de los adultos en la historia de los niños? Y esto es porque han sido, son, y estaría en condiciones de señalar que serán los niños los reflejos de importantes procesos económicos y sociales en Chile, y por qué no, de América Latina. 
Este segundo elemento permite identificar el motivo de la acción, mediante el reconocimiento de sus significados y la observación de actividades concretas en la comunidad, las cuales facilitan la transmisión de la cultura, de manera de generar una protección a contemporáneos y sucesores de agentes que la puedan dañar. Ejemplo de esto es lo mencionado en el siguiente extracto de entrevista:

Por los chiquillos, po', por mis amigos chiquititos, tengo

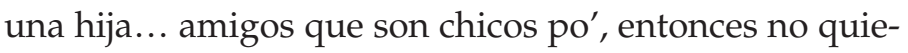
ro... que ellos den un paso al lado, de que ellos no hagan deporte o cosas buenas, porque aquí la droga está aquí, po', en todos lados la weá po', entonces ya los niños van creciendo, van pensando y van viendo cosas que van aprendiendo. (Participante (hombre), El Remanso).

Para la mantención de la cultura, a través de la niñez, los pobladores ejecutan actividades orientadas principalmente a estos. Estas son de tipo lúdico, como "ver películas", o con una orientación económica, como la "feria de las pulgas", en la cual la disposición se focaliza hacia la acumulación de capital, en función del desarrollo de eventos de la propia comunidad.

Por tanto, podríamos señalar que la asignación de significado a la acción y el reconocimiento del motivo de esta permite a los sujetos y colectividades la construcción de estrategias orientadas al fin que sostiene la organización.

Este tipo de estrategias se vinculan con hacer "participar a los niños", lo que permite construir espacios que facilitan romper con la cotidianidad del territorio. Entre estas áreas que se constituyen, se identifican las fiestas religiosas o conmemorativas, reorientadas en función de la niñez, como "fiestas navideñas" y "fiestas patrias". Dichas prácticas comunitarias se identifican en el siguiente extracto de entrevista:

Hacer la feria de las pulgas, pa' tener fondos pa' sus completa's de repente pa' los días viernes, los chiquillos 
tenían data... de repente como una vez al mes pa' ver una película y (...) en las vacaciones, tres días a la semana yo le paso películas a los chiquillos ahí con el data en la sede. (Participante (hombre), El Remanso; entrevista).

Son los sucesores y contemporáneos quienes deben asumir la responsabilidad del objetivo de la comunidad, asignándole significado a su participación para la conservación de la cultura. Esto se da mediante los procesos de socialización que puedan generar los participantes de la Junta de Vecinos con los niños, por sobre el mismo proceso que se ejerce con agentes que puedan dañar la cultura. Esto queda de manifiesto en el siguiente extracto:

... de ahí se empiezan a educar para que puedan pertenecer, no sé po', el día de mañana ellos van a ir madurando, van a querer pertenecer a esto... Yo creo que también hay otros niños que quieren sacar esto adelante". (Participante (hombre), El Remanso; entrevista).

A continuación se presenta un extracto de una entrevista realizada dos meses antes de una actividad navideña.

Por los niños, porque hace cuánto tiempo que no tenemos una navidad, hacen como nueve o diez años, ya ni me acuerdo... que no se hace una navidad (...) Aunque sea poquitito, pero que le sirva a los niños, para que estén contentos, porque por lo menos que estén contentos con un paquetito de dulces (...), con un juguete chico y una bolsita de dulces los niños se sienten más contentos". (Presidenta (mujer), Nueva Patagonia; entrevista).

\section{Dialéctica en la conservación de la cultura}

Si bien ya se ha identificado el significado de la participación como la conservación de la cultura mediante la protección de la niñez, es interesante rescatar su contexto de conservación. Así lo señala Schütz: “...el motivo de una acción no puede comprenderse a menos que se conozca primero el significado de la acción" 
(Schütz, 1993, p. 59). Es decir, una vez que hemos reconocido el significado de la acción, estamos en condiciones de plasmar el motivo de esta.

Existen actividades que se focalizan en la protección de la cultura respecto de agentes internos que la dañan. Estos agentes son identificados como "drogadictos" dentro de la propia comunidad, los cuales han de traer una serie de dificultades para la población. Muestra de esto es el siguiente extracto de entrevista: "Y en cuanto a la droga, pa' qué te voy a decir po', casi está invadido, aquí hay pocos jóvenes que se pueden salvar, porque pa'l lado que tú mirís hay droga" (Participante (mujer), Nueva Patagonia; entrevista).

La conservación de la cultura mediante la participación comunitaria, buscará la rehabilitación o bien la separación del espacio comunitario de estos agentes.

De esta manera, se puede asumir dos tipos de evaluaciones a la hora de proteger la cultura. La primera comprende que estos agentes son parte de la propia cultura y se hace necesario recuperarlos, de manera que se ponen en prácticas recursos orientados hacia la cohesión de la comunidad. Mientras que la segunda forma entiende que estos agentes dañan a la comunidad y que esto es irremediable, por lo tanto se protege a los "niños", ya que son ellos los que presentan una mayor posibilidad de permeabilidad por parte de estos agentes.

En esta dualidad de valoración del agente que daña la cultura existe una relación de intereses opuestos. Esto se debe a que, mientras la organización evalúa y pone en ejecución recursos que se orientan a la mantención de la cultura, los agentes negativos que la dañan configuran y ponen en práctica sus recursos materiales y no materiales que les permiten subsistir dentro de la comunidad.

En estos dos propósitos, la organización pasa a tener un rol fundamental, puesto que facilita la articulación de elementos que permiten alcanzar objetivos, y contiene y protege de agen- 
tes que, pertenecientes a la propia cultura, se constituyen como amenaza.

Para el desarrollo de la organización y el reconocimiento de los significados de participación en la comunidad, se hace necesario un espacio físico que articule las necesidades de la población con los objetivos que sostiene la organización.

Así, el espacio se constituye como un área que suministra el encuentro con otro, facilitando la cohesión y el desarrollo de alternativas para el progreso de la comunidad y de su organización.

De esta manera, estamos en condiciones de señalar que el significado de la participación comunitaria está dado por el reconocimiento particular de los significados de la acción de los sujetos que, mediante un contexto socio-físico-temporal, se pone a disposición de la comunidad, otorgándole un motivo significativo a la acción de esta.

\section{Conclusión}

A partir de lo expuesto, se puede señalar que, para los integrantes o ex integrantes de las organizaciones vecinales, la participación significa tener un mayor o menor grado de involucramiento en las actividades y/o proyectos dirigidos hacia el cuidado de la niñez y la promoción de las condiciones de vida de la comunidad.

Del presente estudio nacen interrogantes para futuras investigaciones, que se orientan hacia la comprensión de las relaciones entre agentes internos y externos que afectan la cultura y a la propia comunidad, y cómo estos constituyen condiciones relativamente óptimas para el desarrollo de la población. O también, por otra parte, ¿cómo influye el progreso de las grandes capitales, en cuanto a autopistas o edificación, en la vida cotidiana en las poblaciones periféricas (colonización del mundo de la vida en la vida cotidiana)? 
Es interesante observar cómo la religión evangélica ha sido un aporte a la propia conservación de la cultura de la comunidad, esto producto de la captación de agentes que afectaban la cultura, los cuales han modificado sus patrones de conducta en función de los ritos del culto evangélico.

Otros elementos que se pueden identificar son temáticas relacionadas con el poder que se constituye al interior de las organizaciones vecinales según género, lo que da espacio para generar reflexiones que se encuentren inscritas en la comprensión de cuál es el papel de la mujer en las organizaciones sociales territoriales.

En el plano metodológico, la investigación presenta un gran desafío epistemológico. La relación del investigador con los informantes ha sido enriquecedora, pero no carente de dificultades como discursos preestablecidos. Bajo esta misma mirada, es importante rescatar el aporte que genera la teoría en la búsqueda del camino metodológico para la captación del contexto significativo de la participación comunitaria.

Finalmente, y no por eso menos importante, es relevante señalar que la presente investigación muestra lo significativo de reconocer las dinámicas, significados e historia de la comunidad para un desarrollo participativo y sustentable, mediante el trabajo en conjunto del sector público, privado y la propia comunidad. De esta manera, nos hemos de focalizar en conseguir un trabajo cooperativo entre estos agentes en función del desarrollo local.

\section{Referencias}

Fundación Junto al Barrio (2011). Encuesta Población El Castillo 2011. Manuscrito en preparación.

Goffman, E. (1991). Los momentos y sus hombres. España: Cultura Libre.

Lutz. B. (2010). La acción social en la teoría sociológica: una aproximación. Argumentos, 23(64) septiembre-diciembre, 199-218.

Martinic, S. (1992). Análisis estructural: Presentación de un método para el estudio de lógicas culturales. Santiago de Chile: CIDE. 
Aproximación al significado en la participación comunitaria. Estudio de caso en la población El Castillo en la diáspora del siglo XXI - Prado

Parsons, T. (1968). La Estructura de la Acción Social II. Madrid: Ediciones Guadarrama.

Pérez, G. (1998). Investigación Cualitativa. Retos e Interrogantes. I, Métodos. Madrid, España: La Muralla.

Salazar, G. (2006). Ser niño "huacho" en la historia de Chile (siglo XIX). Santiago: LOM.

Schütz, A. (1993). La construcción significativa del mundo social. Introducción a la sociología comprensiva. Barcelona: Paidós.

Schütz, A. y Luckmann, T. (2003). Las estructuras del mundo de la vida. Buenos Aires: Amorrortu.

Toledo, M.; Baly, A.; Ceballos, E.; Boelaert, M. y Stuyft, P. (2006). Participación comunitaria en la prevención del dengue: un abordaje desde la perspectiva de los diferentes actores sociales. Revista Social Pública, 48 (1), 39-44.

Weber, M. (1997). Economía y Sociedad. Esbozos de sociología comprensiva. México D.F.: Fondo de Cultura Económica. 